\title{
The AGS with four Helical Magnets
}

\author{
N. Tsoupas, H. Huang, W.W. MacKay, T. Roser and D. Trbojevic
}

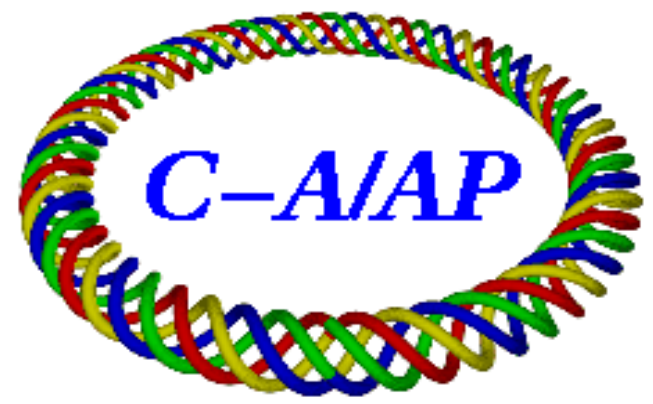

\section{Collider-Accelerator Department Brookhaven National Laboratory Upton, NY 11973}

Notice: This document has been authorized by employees of Brookhaven Science Associates, LLC under Contract No. DE-AC02-98CH10886 with the U.S. Department of Energy. The United States Government retains a nonexclusive, paid-up, irrevocable, world-wide license to publish or reproduce the published form of this document, or allow others to do so, for United States Government purposes. 


\title{
The AGS with four Helical Magnets
}

\author{
N. Tsoupas, H. Huang, W. W. MacKay, T. Roser, and D. Trbojevic
}

\section{Abstract}

The idea [1] of using multiple partial helical magnets was applied successfully to the AGS synchrotron [2], to preserve the proton beam polarization. In this paper we explore in details the idea of using four helical magnets placed symmetrically in the AGS ring. This modification provides many advantages over the present setup of the AGS that uses two partial helical magnets.

First, it provides a larger "spin tune gap" for the placement of the vertical betatron tune of the AGS during acceleration, second, the vertical spin direction during the beam injection and extraction is closer to vertical, third, the symmetric placement of the snakes allows for a better control of the AGS optics, and for reduced values of the beta and eta functions, especially near injection, fourth, the optical properties of the helical magnets also favor the placement of the horizontal betatron tune in the 'spin tune gap", thus eliminating the horizontal spin resonances. In this paper we provide results on the spin tune and on the optics of the AGS with four partial helical magnets, and we compare these results with the present setup of the AGS that uses two partial helical magnets[3].

\section{Introduction}

The preservation of the polarization, of a polarized proton beam, during the acceleration of the beam in a synchrotron is not a trivial task since the polarized beam encounters many "spin- resonances" which may depolarize the beam. The depolarization resonances are classified into imperfection and intrinsic resonances, and an explanation on the nature of these resonances appears in Ref.[2]. In order to overcome these spin resonances which cause depolarization of the polarized beam, an idea was suggested [1] of inserting partial helical magnets in the synchrotron. These partial helical magnets, act either, as strong artificial resonances which minimize the polarization loss at the imperfection resonances, and also eliminate the intrinsic spin resonances. This idea of using multiple helices, was applied to the AGS synchrotron [2] by inserting into the AGS ring two partial helices, and it proved to be successful. Although high field partial helices are beneficial for the preservation of the beam polarization during the beam acceleration, the high field of the helices generate local beam bumps, distort the betatron $\left(\beta_{\mathrm{x}, \mathrm{y}}\right)$ and dispersion $\left(\eta_{\mathrm{x}, \mathrm{y}}\right)$ functions, and also introduce linear beam coupling. These adverse effects, introduced by the partial helices on the beam optics, can be mitigated by the introduction of compensation quadrupoles at the vicinity of the partial helices and by generating local beam bumps at the location of the partial helices [3]. In this paper we further explore the idea [1] by introducing four partial helices, placed symmetrically around the ring. The results of the calculations, of four partial helices, will be compared with those results derived from the calculations of the two partial snakes [3] in the AGS ring. 


\section{Spin Consideration during the acceleration.}

As we mention earlier the function of the partial helical magnets is twofold; first, to introduce an artificial spin resonance which is stronger than the imperfection resonances that occur when the spin tune $v_{\mathrm{sp}}$ of the protons satisfies the condition $v_{\mathrm{sp}}=\mathrm{n}$, and second, to generate a spin tune $v_{\mathrm{sp}}$ during the acceleration cycle such that the condition for the imperfection resonance $v_{\mathrm{sp}}=n \mathrm{n} \pm \mathrm{Q}_{\mathrm{y}},(1)$ is never met during the acceleration cycle. In the expression (1) above, $\mathrm{n}$ is an integer $\mathrm{P}$ is the super-periodicity of the synchrotron and $\mathrm{Q}_{\mathrm{y}}$ is the vertical betatron tune.

In this section we briefly describe the method which we use to derive an expression of the spin tune $v_{\mathrm{sp}}$ as a function of $\mathrm{G} \gamma$, for a proton in a synchrotron with four partial helical magnets. Then we show that by setting properly the vertical tune $\mathrm{Q}_{\mathrm{y}}$, the condition (1) is never satisfied during the acceleration cycle.

To derive an expression of spin tune $v_{\mathrm{sp}}$ as a function of $\mathrm{G} \gamma$ we first calculate the spin rotation matrix $\mathrm{M}$, for a synchrotron with four helical magnets. The spinor's formalism is being used in the derivation.

The following convention is used in the spinor's formalism:

$$
\sigma_{x}=\left(\begin{array}{cc}
0 & 1 \\
1 & 0
\end{array}\right) \quad \sigma_{y}=\left(\begin{array}{cc}
0 & -i \\
i & 0
\end{array}\right) \quad \sigma_{z}=\left(\begin{array}{cc}
1 & 0 \\
0 & -1
\end{array}\right)
$$

The matrices $\sigma_{\mathrm{x}}, \sigma_{\mathrm{y}}$, and $\sigma_{\mathrm{z}}$, correspond to the radial, longitudinal, and vertical direction respectively.

Since the guiding magnetic field in a synchrotron is in the vertical direction, the spin rotation matrix in a section of the synchrotron which bends the beam by an angle $\theta$, is given by:

$\mathrm{M}_{\mathrm{z}}(\mathrm{G} \gamma \theta)=\left\{\mathrm{I} \cos (\mathrm{G} \gamma \theta / 2)-\mathrm{i} \sigma_{\mathrm{z}} \sin (\mathrm{G} \gamma \theta / 2)\right\}$

In equation (3) above I is the identity matrix, $\gamma=\left\{1-(\mathrm{v} / \mathrm{c})^{2}\right\}^{1 / 2}$, and $\mathrm{G}=1.7928$ is the anomalous magnetic moment of the proton.

The spin rotation axis of the partial helical magnets is along the longitudinal beam direction. Therefore the spin rotation matrix which corresponds to a helical magnet is given by:

$\mathrm{M}_{\mathrm{y}}(\delta)=\left\{\mathrm{I} \cos (\delta / 2)+\mathrm{i} \sigma_{\mathrm{y}} \sin (\delta / 2)\right\}$

In equation (4) above $\delta$ is the spin rotation angle about the longitudinal axis.

We assume that the AGS synchrotron contains four partial helical magnets placed symmetrically in the AGS ring. Figure 1 is a schematic diagram of the AGS ring with the four partial helices separated by $90^{\circ}$.

The spin rotation matrix which corresponds to the placement of the partial helices shown in Figure 1, is given by:

$\mathrm{M}(\mathrm{G} \gamma)=\mathrm{M}_{\mathrm{z}}\left(\mathrm{G} \gamma \theta_{5}\right) \mathrm{M}_{\mathrm{y}}(\delta) \mathrm{M}_{\mathrm{z}}\left(\mathrm{G} \gamma \theta_{4}\right) \mathrm{M}_{\mathrm{y}}(\delta) \mathrm{M}_{\mathrm{z}}\left(\mathrm{G} \gamma \theta_{3}\right) \mathrm{M}_{\mathrm{y}}(\delta) \mathrm{M}_{\mathrm{z}}\left(\mathrm{G} \gamma \theta_{2}\right) \mathrm{M}_{\mathrm{y}}(-\delta) \mathrm{M}_{\mathrm{z}}\left(\mathrm{G} \gamma \theta_{1}\right)$

The angles $\theta_{1}$ to $\theta_{5}$ appearing in equation (5), correspond to the angles shown in Figure 1. 
Using expressions (3) and (4) for the rotation matrices $\mathrm{M}_{\mathrm{z}}$ and $\mathrm{M}_{\mathrm{y}}$ one can derive an expression in a closed form, of the spin rotation matrix $M$ as expressed in equation (5). In practice however, we used a computer program to calculate the physical quantities of the spin tune $v_{\mathrm{sp}}$, and of the vertical spin direction $\mathrm{S}_{\mathrm{z}}$ at the Injection point where the beam is injected with energy corresponding to the value of $\mathrm{G} \gamma=4.5$ and at the extraction point where the beam is extracted from AGS with beam energy corresponding to the value of $\mathrm{G} \gamma=45.5$.

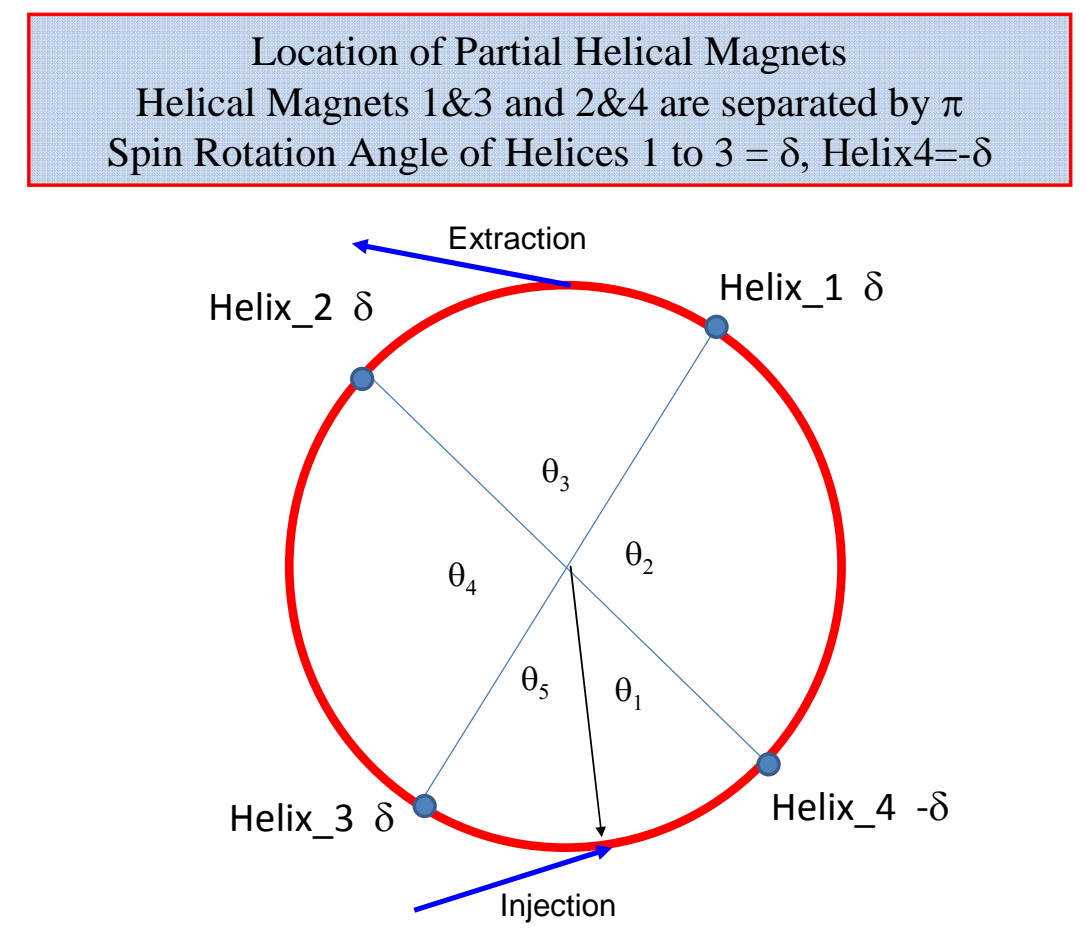

Figure 1. Schematic diagram of the AGS ring with four Helices symmetrically placed around the ring.

The spin tune $v_{\mathrm{sp}}$ is derived from the expression:

$$
\cos \left(\pi v_{s p}\right)=\frac{1}{2} \operatorname{Tr}\{M(G \gamma)\}
$$

In the expression (6) above, $\operatorname{Tr}\{\mathrm{M}(\mathrm{G} \gamma)\}$ is the trace of the spin-rotation matrix $\mathrm{M}$ in equation (5).

The stable spin direction $\overrightarrow{\vec{b}}$ at injection ( $\mathrm{G} \gamma=4.5)$ and at extraction ( $\mathrm{G} \gamma=45.5)$ is given by the expression:

$$
\overrightarrow{\vec{b}}=\left[\frac{i}{2 \sin (\phi / 2)}\right] \operatorname{Tr}(\bar{\sigma} M) \quad \text { (7) } \quad \text { where: } \quad \cos \left(\frac{\phi}{2}\right)=\frac{1}{2} \operatorname{Tr}(M)
$$


The spin tune $v_{\mathrm{sp}}$ is calculated using the expression (6) and is plotted as a function of $\mathrm{G} \gamma$ in Figure 2, for various strengths (spin rotation angles) of the partial helices. The black trace in the plot shown in Figure 2, corresponds to the spin tune generated by the two partial helices $[1,2]$ which are presently installed in the AGS. The spin rotation angle of one of the partial helices is $19.08^{\circ}$ or $(10.6 \%)$ and that of the second helix, which is located in the ring $120^{\circ}$ away from the first helix, is $15.48^{\circ}$ or (8.6\%). The other three traces (red blue and green) correspond to the spin tune when the AGS has four partial helices separated azimuthally by an angle of $90^{\circ}$. The corresponding strengths of the helices are $8.06 \%$ (red) 10\% (blue) and 11.098\% (green). At higher values of $\mathrm{G} \gamma$ than those indicated in the graph, the spin tune repeats itself with one of the periods shown in the graph. .

When the value of the fractional betatron tune $\mathrm{Q}_{\mathrm{y}}$ is higher than the fractional value of the spin tune $v_{\mathrm{sp}}$, the condition for an intrinsic spin resonance, $v_{\mathrm{sp}}=\mathrm{nP} \pm \mathrm{Q}_{\mathrm{y}}$, will not be satisfied, therefore no intrinsic spin resonance will occur during the acceleration cycle. The above statement is true for the case of the two partial helices as shown by the spin tune represented by the black curve. However in the case of four helices, the values of the spin tune allows for a "wider spin tune gap" where the betatron tunes can be placed, thus providing a larger range for the placement of the betatron tunes, even at lower strength of the helices.

\section{Spin Tune in AGS vs $\mathrm{G} \gamma$}

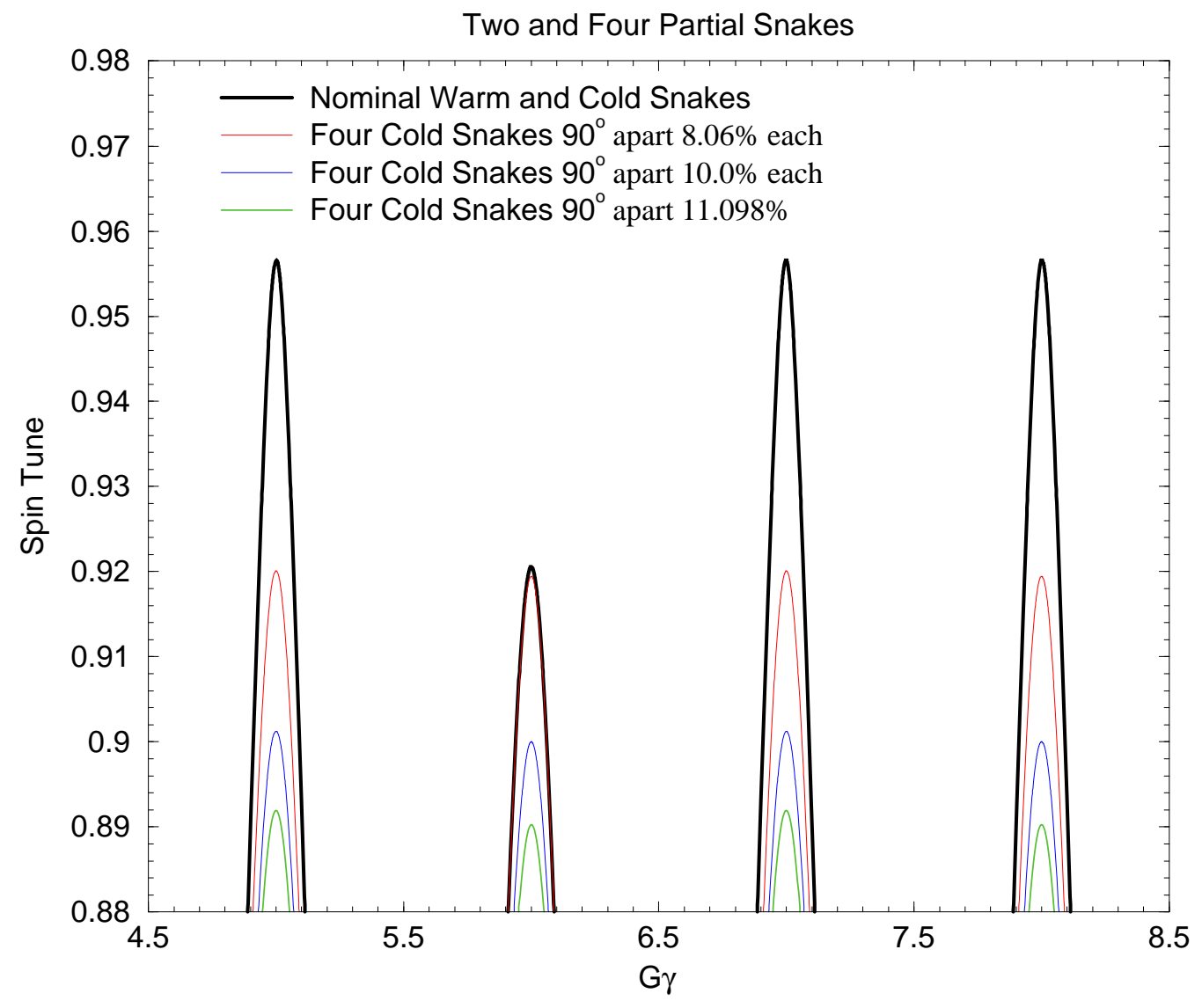


Figure 2. The spin tune $v_{\mathrm{sp}}$ in AGS as a function of $\mathrm{G} \gamma$. The black trace corresponds to the spin tune when the AGS has two partial helices separated azimuthally by an angle of $120^{\circ}$, with $10.0 \%$ and $8.6 \%$ strength respectively for each helix. The other traces (red blue and green) correspond to the spin tune when the AGS has four partial helices separated azimuthally by an angle of $90^{\circ}$. The corresponding strengths of the helices are 8.06\% (red) 10\% (blue) and 11.098\% (green). At higher values of $\mathrm{G} \gamma$ than those indicated in the graph, the spin tune repeats itself with one of the periods shown in the graph.

Another issue, related to the spin, when four helical magnets are placed in the AGS, is the stable spin direction at the injection and extraction points of the AGS. Since there are no spin rotators at either, the injection or extraction transport lines, it is desirable that the stable spin direction in AGS at the injection or extraction points to be as close to the vertical as possible in order to "match" the required stable spin direction of the injection or extraction transport line, which is vertical. (Not very true for extraction but let's not make an issue out of it).

Table 1 . below, the $2^{\text {nd }}$ row contains values of the spin directional cosines with the vertical at the injection point $\left(2^{\text {nd }}\right.$ column $)$ and the extraction point $\left(3^{\text {rd }}\right.$ column $)$, for the case of two helices, and the $3^{\text {nd }}$ row contains values of the spin directional cosines with the vertical at the injection point $\left(2^{\text {nd }}\right.$ column $)$ and extraction point ( $3^{\text {rd }}$ column $)$, for the case of four helices.

Table 1 . The values of the spin directional cosine with the vertical, at the injection point $\left(2^{\text {nd }}\right.$ column $)$ and extraction point ( $3^{\text {rd }}$ column $)$, for the case of two helices $\left(2^{\text {nd }}\right.$ row $)$ and the case of four helices ( $3^{\text {rd }}$ row).

\begin{tabular}{|l|c|c|}
\hline & Dir_cos S & Dir_cos S \\
& Inject. SS_L20 G $\gamma=4.5$ & Extr. SS_H10 G $\gamma=45.5$ \\
\hline $\begin{array}{l}\text { Two Helices } \\
\text { A20 and E20 }\end{array}$ & 0.9979 & -0.9763 \\
\hline Four Helices & 1.0000 & -0.9828 \\
\hline
\end{tabular}

\section{Beam Optics Consideration of the AGS synchrotron with four Helices.}

A challenging task of introducing helices into a medium energy synchrotron is to minimize the effect of the relatively high field helical magnets on the beam optics of the circulating beam. This effect is especially large, at injection energy where the beam rigidity is low and the size of the beam is large. In this section we describe the beam optics of the circulating beam in AGS at injection, and discuss a possible method to ameliorate the effect of the helical magnet on the circulating beam. The required constrains on the beam parameters, dispersion functions and betatron tunes, during the beam acceleration, will also be discussed.

The AGS synchrotron consists of 240 combined function magnets and has a superperiodicity $\mathrm{P}=12$, with 20 magnets per superperiod. Two Quadrupoles, one located at the 
straight section SS03 ant the other at SS17 of every superperiod, are used to adjust the horizontal and vertical betatron tunes respectively. Figure 3 shows the beta $\left(\beta_{\mathrm{x}, \mathrm{y}}\right)$ and eta $\left(\eta_{x, y}\right)$ functions of the circulating beam in a closed orbit, over three superperiods of the Bare-AGS, where the tune quadrupoles are set at zero excitation. The corresponding tunes for a bare AGS, at Injection energy are shown in columns 2 and 3 of Table 2.

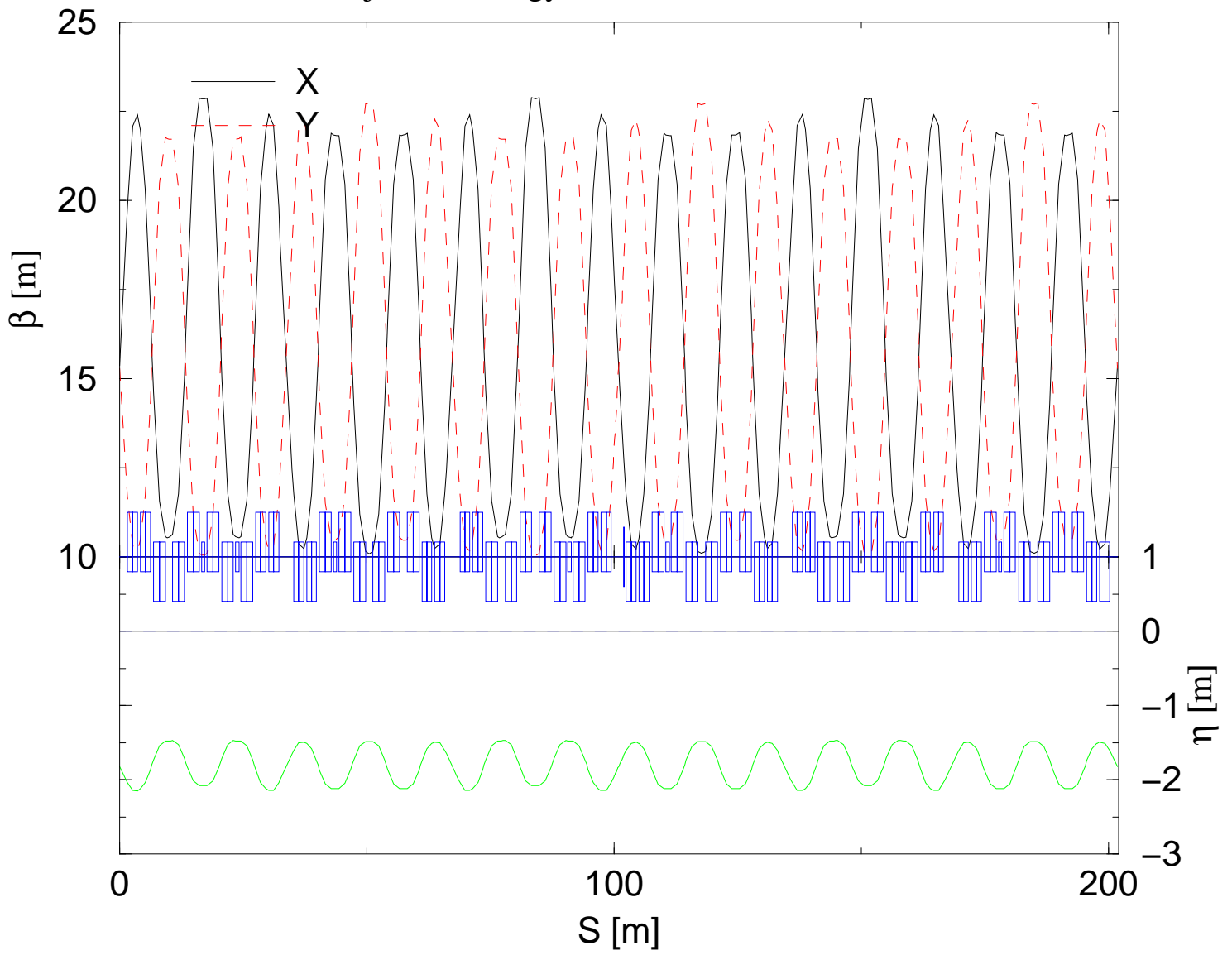

Figure 3. The beta $\left(\beta_{\mathrm{x}, \mathrm{y}}\right)$ and eta $\left(\eta_{\mathrm{x}, \mathrm{y}}\right)$ functions over three super-periods of the bare AGS. The horizontal and vertical tune qudrupoles (one of each in each super-period) are set to zero. The corresponding betatron tunes appear in the $2^{\text {nd }}$ column of Table 2 .

Table 2. The horizontal and vertical betatron tunes $\left(\mathrm{Q}_{\mathrm{x}}, \mathrm{Q}_{\mathrm{y}}\right)$ for the AGS at various settings as they appear in the $1^{\text {st }}$ column.

\begin{tabular}{|c|c|c|}
\hline & $\mathrm{Q}_{\mathrm{x}}$ & $\mathrm{Q}_{\mathrm{y}}$ \\
\hline Bare_AGS & 8.7127 & 8.7675 \\
\hline $\begin{array}{c}\text { Bare_AGS } \\
\text { 4(Cold Helices) }\end{array}$ & 9.1864 & 8.9884 \\
\hline $\begin{array}{c}\text { Non-Bare-AGS } \\
\text { 4(Cold Helices) }\end{array}$ & 8.9785 & 8.9874 \\
\hline
\end{tabular}


The insertion of four helical snakes symmetrically placed around the AGS ring, reduces the super-periodicity of the AGS to four. Figure 4 shows the beta $\left(\beta_{\mathrm{x}, \mathrm{y}}\right)$ and eta $\left(\eta_{\mathrm{x}, \mathrm{y}}\right)$ functions of the circulating beam in closed orbit, over three superperiods of the bareAGS, with a helical magnets placed at the center of the three superperiods. The corresponding tunes for a bare AGS, at Injection energy are shown, in columns 2 and 3 of the $3^{\text {rd }}$ row of Table 2 . Here we assume that the focusing properties of each of the four helices are identical. Although this maybe valid for the three helices which rotate the spin by an angle $+\delta$, it may not be true for the fourth helix which rotates the spin by an angle $\delta$. This assumption requires further investigation.

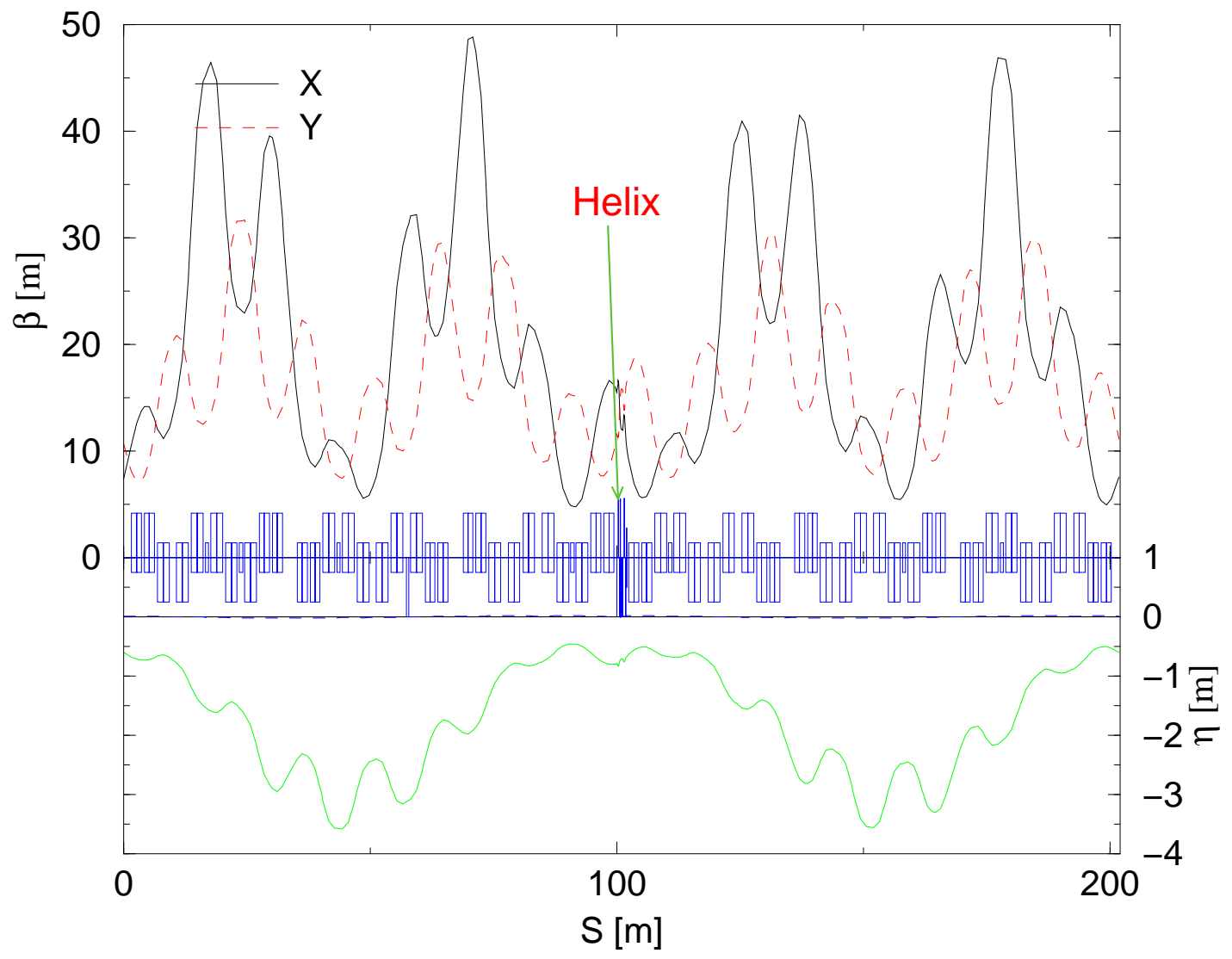

Figure 4. The beta $\left(\beta_{\mathrm{x}, \mathrm{y}}\right)$ and eta $\left(\eta_{\mathrm{x}, \mathrm{y}}\right)$ functions over three super-periods of the bare AGS with a helical magnet inserted at the middle, as shown by the green arrow in the figure. The horizontal and vertical tune qudrupoles (one of each in each super-period) are set to zero. The corresponding betatron tunes appear in Table 2.

Although the insertion of the helix distorts the beam optics of the AGS, the beam is still stable, and the betatron tunes can be set at the required values with the use of the tune quadrupoles. It is desired however to reduce the values of the beta and dispersion function and provide more control in the AGS during the beam acceleration. This can be accomplished by the insertion of thin quadrupoles in the straight section at the vicinity of 
the helices, as well as by connecting floating power supplies across existing quadrupoles like the tune quadrupoles. The effect of such compensation quads on the beta $\left(\beta_{x, y}\right)$ and eta $\left(\eta_{x, y}\right)$ functions, is shown in Figure 5 which shows the beta $\left(\beta_{x, y}\right)$ and eta $\left(\eta_{x, y}\right)$ functions of the circulating beam in closed orbit, over three superperiods of the AGS, with a helical magnets placed at the center of the three superperiods. The beta $\left(\beta_{\mathrm{x}, \mathrm{y}}\right)$ and eta $\left(\eta_{x, y}\right)$ functions shown in Figure 5 can be compared with those shown in Figure 4. The relative location and the strength of the compensation quads appear in Table 3 . The betarton tunes which correspond to the beam optics shown in Fig. 5 have been adjusting by using the tune quadrupoles, and the values of the tunes appear in Table 2.

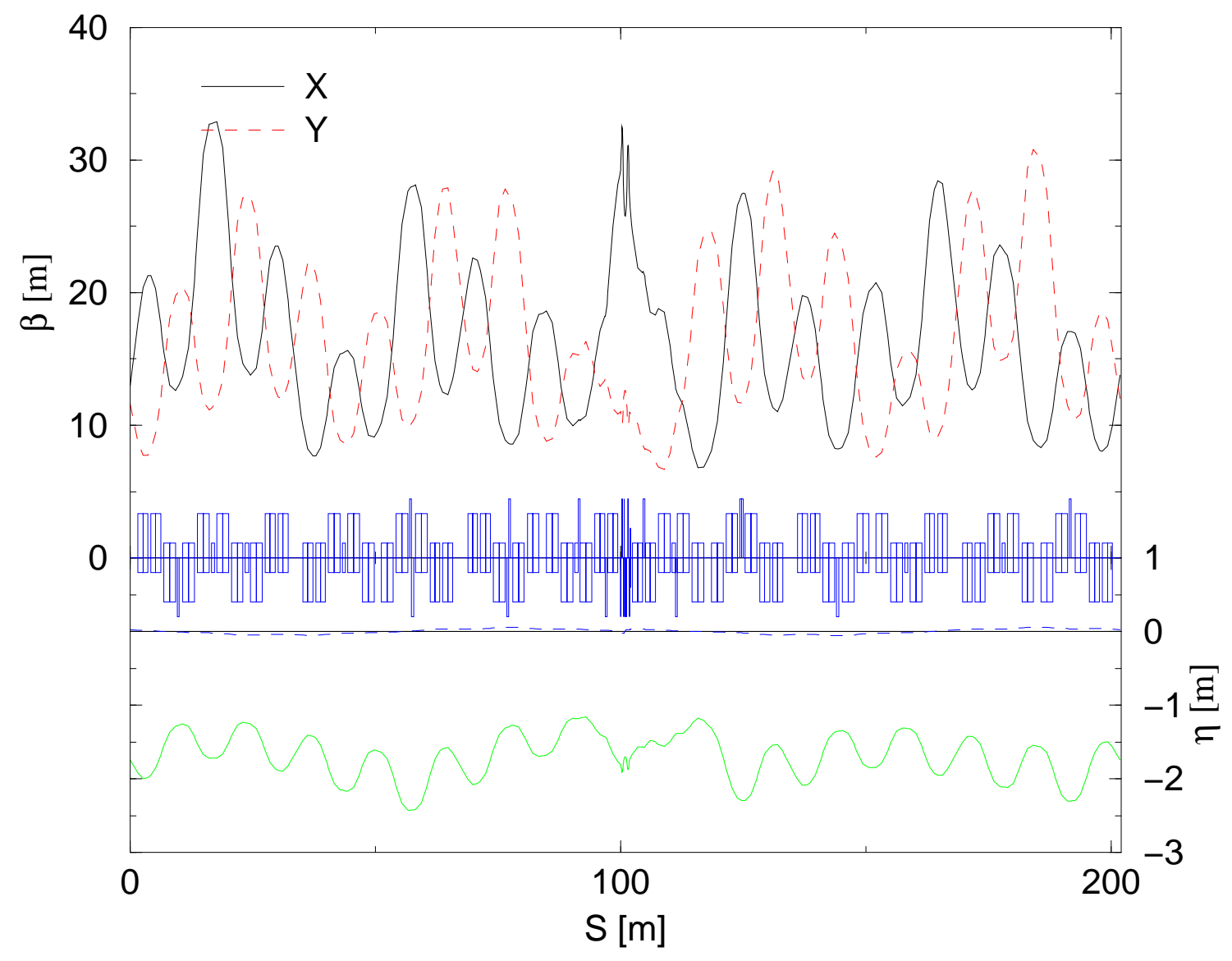

Figure 5 . The beta $\left(\beta_{\mathrm{x}, \mathrm{y}}\right)$ and eta $\left(\eta_{\mathrm{x}, \mathrm{y}}\right)$ functions over three super-periods of the bare AGS with a helical magnet inserted at the middle. A number of compensation quads (see Table 3) has been inserted in various straight sections to help reduce the values of the beta and eta functions as compared to those shown in Figure4. The horizontal and vertical tune qudrupoles have also been adjusted to provide the required betatron tunes which appear in Table 2. 
Table 3. The location of the compensation quadrupoles relative to the helix, and their strength in $\mathrm{m}^{-2}$.

\begin{tabular}{|c|c|c|c|c|c|c|}
\hline 1st SupPer. & \multicolumn{5}{|c|}{$2^{\text {nd }}$ Superperiod } & $3^{\text {rd }}$ SupPer. \\
\hline CQ1 & CQ2 & CQ3 & Helix & CQ4 & CQ5 & CQ6 \\
\hline SS_03 & SS_07 & SS_09 & SS_10 & SS_11 & SS_13 & SS_17 \\
\hline 0.0405 & -0.3031 & 0.3201 & & -0.4707 & 0.1594 & 0.0031 \\
\hline
\end{tabular}

\section{Further studies, investigations, and suggestions}

a) Study the interference of the Extraction beam bumps of AGS with the Helices placed symmetrically in AGS.

b) Generate local beam bumps at the location of each of the Helices.

c) Maximum field of each helix should not exceed the $2.4 \mathrm{~T}$, this may allow for an increased aperture of the helical magnet.

d) Install horizontal corrector magnets at the entrance and exit of each helix.

e) Calculate the focusing properties of the "left-handed" vs "right-handed" helices.

f) Investigate the beam optics of the AGS with four helical magnets but with two fold symmetry.

g) Other....

*Work supported by the US Department of Energy

[1] T. Roser, et al., Proc. EPAC04, (2004), p. 1577

[2] H. Huang, et al., PRL 99, 154801(2007)

[3] N. Tsoupas et. al. CAD-391 Internal Report 